(Aus dem physiologischen Institut der Königlichen Universität Breslau.)

\title{
Beiträge zur Kenntniss der Säurebildung und des Stoffumsatzes im quergestreiften Muskel.
}

\author{
Von \\ Emil Gotschlich, \\ Assistent am $\mathrm{kgl}$. hygienischen Institut.
}

\section{Vorbemerkung.}

Nachstehende Abhandlung entstand auf Anregung von Herrn Geheimrath Heidenhain und wurde in seinem Institnte unter seiner speciellen Leitung ausgeführt. Einen grossen Theil der im Folgenden zu besprechenden Fragen hatte bereits Herr Geheimrath Heidenhain selbst im Herbste 1892 einer Bearbeitung unterzogen und gestattete mir gütigst einen Einblick in seine Resultate. Hierfür, sowie für die mehrfache freundliche Aufnahme auf seinem Institut, sei es mir vergönnt, an dieser Stelle meinem bochrerehrten Lehrer meinen bescheidenen Dank za sagen.

\section{Einleitung und Methodik der Säurebestimmung.}

Seit Du Bois-Reymond's (1) 1) Entdeckung der Reaktion des ruhenden, thätigen und abgestorbenen Muskels sind viele Untersuchungen auf diesem Gebiete angestellt worden. Dieselben waren jedoch meist von vorwiegend chemischem Interesse, indem sie entweder die Natur und Entstehungsweise desjenigen Körpers zu ermitteln suchten, welcher die saure Reaktion des thätigen oder abgestorbenen Muskels bewirkt, oder indem sie, besonders neuerdings, sich der Frage zuwandten, ob nicht auch der ruhende Muskel Säure enthalte. So viel schätzbares Material auch diese Arbeiten zu Tage förderten, so ist doch auf diesem Gebiete noch

1) Die Zahlen beziehen sich auf das im Anhang beigegebene Literaturverzeichniss. 
keine Einigkeit geschaffen; im Gegentheil stehen sich, gerade in den neveren Arbeiten, schroff die Ansichten gegenüber, so dass Heffter (2) vor kurzem zu dem Ausspruch kommen konnte: „So haben die mittelst Indicatoren studirten Veränderungen des Muskels bei der Thätigkeit und Starre für die Erkennung der dabei stattfindenden chemischen Untersuchungen ${ }^{1}$ ) gegenwärtig wenig Werth, weil sie keine sicheren Schlïsse zu ziehen gestatten."

Abgesehen aber von dieser rein chemischen Seite der Frage besteht noch eine andere, die hohen physiologischen Interesses werth erscheint; die saure Reaktion des Muskels lässt sich als ein Mass seines Stoffumsatzes auffassen; man kann daher dureh Untersuchung der Säuerung unter verschiedenen Versuchsbedingungen Anhaltspunkte zur Erkenntniss des Stoffumsatzes selbst unter diesen Bedingungen gewinnen und so für die Kenntniss des inneren Mechanismus der Muskelthätigkeit einen Beitrag erhalten. In dieser Richtung sind jedoch bisher nur wenige Forscher vorgedrungen. So bestätigte Heidenhain (3) auf diesem Wege sein bereits durch thermoelektrische Versuche gewonnenes Resultat, dass Spannung den Stoffumsatz des thätigen Muskels vermehrt; so untersuchten ferner seine Schuler Landau und Pacully (4) die Säuerung des Muskels unter verschiedenen mechanischen Bedingungen während des Erschlaffungsprocesses; später ermittelte Ranke (5) durch titrimetrische Säurebestimmungen das unveränderliche Säurebildungsmaximum des Muskels; endlich bestätigte Gross (6) durch die Untersuchung der Reaktion des Muskels das Resultat früberer Autoren, dass das Nervensystem dureh sein Absterben die Todtenstarre beschleunigt. Der Grund, weshalb Untersuchungen in der angedeuteten Richtung nur in spärlicher Zahl vorhanden sind, liegt wohl hauptsächlich darin, dass der Forschung zar Erkenntniss des Stoffumsatzes im Muskel eine andere, weit vorzüglichere Methode za Gebote steht, nämlich die thermoelektrische Bestimmung der Wärmeentwicklung im thätigen Muskel; und in der That sind auf diesem Wege dureh zahlreiche Arbeiten, insbesondere von Heidenhain, Fick und ibren Sehülern und erst neuerdings von M etzuer (7) wichtige Resultate erzielt worden. Der Vorzug dieser Methode vor der Säure-Bestimmung liegt sowohl in ihrer quantitativen Präcision, als auch

1) Soll wohl "Umsetzungen" lreissen. 
vor allem darin, dass sie die störenden individuellen Differenzen durch Ausfübrung zahlreicher Versuche an demselben Präparat fast vollkommen auszuschalten vermag; die Säurebestimmung lässt sich dagegen an einem Maskel natürlich immer nur einmal machen. Allerdings hat in neuester Zeit Landsberger (8) versucht, diese Fehlerquelle zu umgehen; statt, wie bisher alle anderen Autoren, den Muskel bei der Säurebestimmung zu vernichten, lässt er ihn unverletzt und bestimmt die in das flüssige Medium diffundirte Säuremenge, was sich allerdings an einem Präparat mehrmals ausfiuhren lässt; indessen stehen der allgemeineren Anwendung dieser Versucbsanordnung wohl die erheblichsten Bedenken entgegen, indem einerseits die Fixirnng des Muskels in einem bestimmten Zustande unmöglich ist und andererseits, wie Landsberger selbst betont, die Diffusion nur sehr langsam vor sich geht.

Wenn wir es nun, trotz aller dieser Bedenken, unternehmen, mittelst quantitativer Säurebestimmungen die Grösse des Stoffumsatzes im Muskel unter gewissen Bedingungen erforschen za wollen, so ermuthigt uns einerseits hierzu der Parallelismus zwischen Säurebildung und Wärmeentwicklung des thätigen Muskels, welcher in den Versuchen von Heidenhain (3) unter bestimmten Versuchsbedingungen nachgewiesen wurde, und andererseits erscheint es von hohem Interesse, nachzuforschen, ob dieser Parallelismus auch unter anderen Bedingungen fortbesteht. Ausserdem aber bietet die Säurebestimmung unter gewissen Verhältnissen direkte Vortheile vor der thermoelektrischen Massmethode, nämlich dann, wenn es gilt, eine Erhöhung des Stoffumsatzes im Muskel nacbzuweisen, die in der Zeiteinbeit minimal ist und erst bei fortgesetzter längerer Dauer einen erheblichen Gesammtwerth erreicht; es findet dann eine Summation der fort und fort gebildeten kleinsten Säuremengen im Muskel statt, so dass schliesslich eine chemisch nachweisbare Gesammtmenge von Säure entsteht, während die gleicbzeitig gebildeten minimalen Wämemengen stets sofort nach aussen abgegeben werden und sich so, trotz der grösseren absoluten Empfindlichkeit der thermoelektrischen Methode, dem Nachweis entziehen. Gerade dieser Fall ist aber, wie wir sehen werden, in mehreren unserer Versucbsreihen verwirklicht. - Zur Charakterisirung aller unserer Versuche ist hervorzuheben, dass uns die Säuerung des Muskels nur insoweit interessirt, als sie eine Differenz des Stoff- 
umsatzes zweier Muskeln zu erkennen und eventuell zu messen gestattet; die qualitativ-chemische Natur dieser Differenz, also die Fragen, ob es sich um freie Säuren oder saure Salze handle etc., liegen durchaus ausser dem Bereich unserer Betrachtungen. Wir bedienten uns daher zur Säurebestimmung nicht etwa der chemischen Analyse, sondern einfach des Vergleichs der Reaktion zweier Muskeln auf einen Indicator. Eine neue, früher ungeahnte Complication hat freilich jede auf diesem Wege angestellte Säurebestimmung durch die Untersuchungen von $\mathrm{R} \ddot{o} \mathrm{~h} \mathrm{~m}$ a n $\mathrm{n}(9)$ und Sp it zer (10) über die Indicatoren erhalten; derselbe Muskel reagirt auf verschiedene Indicatoren verschieden, und die Substanz, welche den Indicator beeinflusst, braucht durchaus nicht mit der vom Muskel gebildeten Substanz identisch zu sein. Z. B. könnte die im Muskel etwa entstehende freie Milchsäure zur Bildung saurer Phosphate führen, die ihrerseits erst den Indicator beeinflussen. Auch von allen diesen Fragen abstrahiren wir hier grundsätzlich, und wo wir im Folgenden das Wort „Säurebildung،" gebrauchen, wollen wir damit nichts iber die Natur dieses Vorganges präjudiciren, sondern nur die einfache Thatsache bezeichnen, dass unter den bestimmten Versuchs-Bedingungen gewisse Verschiebungen der chemischen Affinitäten in der Muskelsubstanz sich vollzogen haben, die ibren Ausdruck in der Farbenveränderung des Indicators finden. Die Existenz und die Grösse dieser Verschiebungen aber ist ein unmittelbarer Ausdruck einer chemischen Arbeit, eines veränderten Stoffumsatzes.

Hiernach schreiten wir zur specielleren Mittheilung unserer Versuchsanordnung. Wir verwendeten stets den Gastrocnemius des Frosches. Nach schneller, schonender Präparation wird de̊r Muskel durch zartes Betupfen mit Fliesspapier von Blut und Lymphe gereinigt und dann zum Versuche verwendet; der Controlmuskel verweilt inzwischen in der feuchten Kammer. Selbstverständlich ist jede mechanische Misshandlung des Muskels peinlichst zu vermeiden. Muskeln mit subfascialen Blutergïssen, wie sie so häufig vorkommen, sind unbrauchbar, da das alkalische Blut die saure Reaktion beeinträchtigt. Ebenso sind Muskeln, die spontan flimmern und zucken, sowie solehe, die von vornherein auf den Indicator saner reagiren, unverwendbar. 'Nur grosse und muntere Frösche sind zu den Versuchen zu wählen, wenn man positive und einigermassen iibereinstimmende Resultate erhalten will. 
Nach gehöriger Vorbereitung des Präparates folgt nunmehr der Versuch, dessen Anordnung natürlich in den verschiedenen Reihen sehr verschieden war und daher erst im speciellen Theil dieser Arbeit geschildert werden kann.

Nach Beendigung des experimentellen Eingriffs gilt es nun, den Zustand des Muskels möglichst schnell zu fixiren und die in ihm enthaltene Säuremenge möglichst vollständig zu gewinnen. Beides leistet die von $\mathrm{H}$ e i d e $\mathrm{n} \mathrm{h}$ a in (3) angewandte Behandlung des Muskels mit gesättigter Kochsalzlösung. Der Muskel wird also, nachdem er rasch zwischen zwei tarirten Uhrschälchen abgewogen war, in einen Porzellanmörser gebracht nud mit $2 \mathrm{~cm}$ gesättigter Kochsalzlösung mittelst eines Porzellanpistills zerquetscht; das erhaltene Extract wird durch entfettete Watte filtrirt und in einem kleinen graduirten Masscylinder gesammelt. Diese Procedur wird noch zweimal, wiederum mit je $2 \mathrm{cem}$, wiederholt, so dass im Ganzen $6 \mathrm{ccm}$ Extract vorhanden sind, von denen uns allerdings wegen der unvermeidlichen Verluste beim Filtriren ete. nur $5 \mathrm{ccm}$ zur weiteren Verwendung bleiben. Demselben Verfahren wird der Controlmuskel unterworfen; selbstverständlich werden dazu andere Gefässe verwendet. Von den gesammelten Mengen beider Extracte werden genau gleiche Mengen zur Untersuchung verwendet. Sämmtliche bei der Extraction gebrauchten Gefässe müssen natürlich vorher einer peinlich sorgfältigen Reinigung unterworfen werden, da schon geringe Spuren von Alkali oder Säure, die ihnen etwa anhaften, das Resultat beeinträchtigen können; die Gefässe wurden zuerst in fliessendem Wasser energisch ausgewaschen, dann mit destillirtem Wasser nachgespült und schliesslich mit einem reinen leinenen Tuche ausgetrocknet. Noch ist darauf zu achten, dass der Muskel in der gesättigten, ihn stark reizenden Kochsalzlösung nicht zuckt und dadurch neue Säure entwickelt; man muss daher sofort mit dem Pistill bei der Hand sein.

Die zur weiteren Untersuchung bereiteten Extracte sind ziemlich klar, durchscheinend, schwach gelblich, opalescent. Eine starke gelbe Eigenfarbe der Extracte, sowie ein starker Unterschied in der Eigenfarbe beider macht sie zur Säurebestimmung ungeeignet. Welchen Indicator sollen wir nun wählen? Da Bois - Rey mond (1), Heidenhain (3), Landau und Pacully (4), Ranke (5) verwendeten Lakmus, letzterer sowie Röhmann (9) auch Curcuma, Röhman ausserdem noch Lakmoid, Dreser (11) Säurefuchsin, 
Moleschot und Battistini (12), Landsberger (17) und Meyerhold (13) Phenolphthalein, Heffter (2) Phenolphthalein und Rosolsäure. Wir bedienten uns auf Anrathen von Herrn Professor Röhmann des Alizarinnatriums, eines Farbstoffs, welchen dieser Autor (14) zur Prüfung der Reaktion des elektrischen Organes des Zitterrochens angewendet hatte. Die Einführung dieses neuen Indicators rechtfertigt sich durch seine ungemein grosse Empfindlichkeit; schon auf die im gewöhnlichen destillirten Wasser enthaltenen Kohlensäuremengen reagirt er merklich. Die Farbe des Indicators ist in alkaliseher Fliussigkeit tief blau- bis roth-violett, in saurer bellgelb. Zur Verwendung in unseren Versuchen wurde die Lösung des Indicators folgendermassen bereitet: Es wird eine iibersättigte Lösung von trockenem gepulverten Alizarin (Dr. Grubler) in 1/100 Normal-Natronlauge bereitet und eine kleine Menge hiervon mit derselben Lauge zehnfach verdünnt; die'so entstehende Flüssigkeit muss im Dunkeln wohl verschlossen aufbewahrt and öfters erneuert werden, da sie sich durch Einwirkung der atmosphärischen Kohlensäure leicht zersetzt. 5 Tropfen dieser Flüssigkeit werden dem 5 cem Extract hinzugefügt, wodurch das Extract des ruhenden Muskels schön roth-violett bis roth, das des tetanisirten Muskels gelb gefärbt wird. In unseren Versuchsreihen bandelte es sich freilich oft um viel kleinere Säuremengen; dieselben gaben sich dann dadurch kund, dass das betreffende Extract in der continuirlichen Scala der Farbennuancen (violett, rothviolett, roth, orange, röthlichgelb, reingelb) von violett bis gelb dem letzteren näher stand. Behrufs dieser oft schwierigen Vergleichung wurden die beiden Messröhrchen gegen einen weissen, vom Tageslicht bell beleuchteten Untergrund dicht neben einander gehalten; wir bedienten uns also in unsern Versuchen der colorimetrischen Methode, welche zur qualitativen Feststellung der Muskelreaktion zuerst von He ide nhain (3) angewendet worden ist.

Nun aber gilt es, den qualitativ festgestellten Säuerungsunterschied auch quantitativ auszuwerthen, wie dies unseres Wissens zuerst von $\mathrm{Ranke}(5)$ auf titrimetrischem Wege geschehen ist. Auch wir wendeten dieses Verfahren an, indem wir zu dem saueren Extracte tropfenweise so lange $1 / 100$ Normal-Natronlauge hinzufügten, bis die Farbendifferenz beider Extracte völlig ansgeglichen war. Beim Umschütteln wurden die Röhrehen nicht mit dem 
Daumen, sondern mit wohl gereinigten Korken verschlossen. Die Bürette; welche die Natronlauge enthielt, war durch ein aufgesetztes, mit Natronkalk gefülltes Rohr vor dem Zutritt der atmosphärischen Koblensäure geschiitzt. Die Menge Alkali, welche erforderlich ist, um die Farbendifferenz beider Extracte auszugleichen, ist ein unmittelbares Mass für die durch den experimentellen Eingriff bewirkte Säuernng im Muskel. Nun lässt sich aber einwenden, dass möglicherweise Körper mit freien sauren Affinitäten im Muskel vorhanden sind, die sich in unserer Extractionsflüssigkeit nicht lösen, oder Umsatzprodukte geschaffen wurden, welche die Farbe unseres Indicators nicht beeinflussen. Wir anerkennen diese Möglichkeiten und geben zu, dass unserem Nachweise vielleicht ein Theil des Stoffumsatzes im Muskel entgeht; da aber unser Verfahren in allen Versuchen genau dasselbe war, so entfällt stets derselbe Theil unserer Beobachtung, und wir haben daher immer noch in unserer Säurebestimmung ein proportionales, wenn auch kein äquivalentes Mass für den gesammiten Stoffumsatz. Nur dann, wenn anch das Verhältniss der durch unsere Säurebestimmung erkennbaren Umsatzprodukte zu denen, die unserem Nachweise entgehen, bei verschiedenen Versuchs-Bedingungen, z. B. bei verschiedener Reizstärke, sich änderte, gäbe unser Verfahren überhaupt kein Mass des Gesammtstoffumsatzes; dass aber dieser Einwand im Allgemeinen nicht zutrifft, beweisen eben unsere positiven Resultate; höchstens könnte er zur Elklärung mancher Unregelmässigkeiten herangezogen werden.

Schliesslich haben wir, mit Riicksicht auf die Bedeutung, welche die Lebre von den Indicatoren seit den Arbeiten von Röhmann (9) und Spitzer (10) für physiologische Versuche gewonnen hat; auch die chemischen Eigenschaften unseres Indicators, des Alizarinnatriums, einer eingehenden Prüfung unterzogen. Die Mittheilung der Resultate dieser Versuche wïrde den Rahmen dieser Arbeit weit überschreiten; wir gedenken dieselbe demnäehst in einer eigenen Abhandlung zu veröffentlichen. Hier sei nur erwähnt, dass sich der Indicator seinem chemischen Verhalten nach als ungemein brauchbar für unsere augenblicklichen Zwecke erwies, insbesondere wegen seiner grossen Empfindlichkeit gegen saure Salze.

Nachdem wir im vorigen unser Versuchsverfahren im einzelnen Falle hinlänglich geschildert haben, bleibt nun noch übrig, 
anzugeben, wie wir dieses ans Einzelfällen bestehende Rohmaterial weiter verwendet haben. $\mathrm{Zu}$ diesem Zweck wurden die einzelnen Fälle durch Reduction auf gemeinsame Einheiten vergleichbar gemacht. Die Säuerung wurde in allen Versuchen auf mgr Milchsäure berechnet, womit selbstverständlich nicht behauptet werden soll, es habe sich um diese allein gehandelt; $1 \mathrm{ccm}$ unserer $1 / 10 \mathrm{n}^{-}$ Normal-Natronlauge entsprach $0,9 \mathrm{mgr}$ Milchsäure. Die so berechneten Säurewerthe wurden auf das einheitliche Muskelgewicht von $1,0 \mathrm{gr}$ bezogen. In solchen Versuchsreihen, wo eine Relation zwischen der Grösse der Arbeitsleistung und Säurebildung des Muskels aufgestellt werden sollte, musste natürlich auch die erstere in einheitlichem Masse, grmm (Grammmillimeter), berechnet und auf die Gewichtseinheit des Muskels reducirt werden; endlich war es bier auch erforderlich, die Säurebildung auf eine Arbeitseinheit zu beziehen, als welche wir in verschiedenen Tabellen 10000 oder 100000 grmm angenommen haben. Hiernach ist die Einrichtung unserer im Anhang beigebenen Tabellen ohne weiteres verständlich. Wir geben in ihnen aber nicht bloss die mehrfachen Reductionen unterworfenen und daher für sich allein wenig instruktiven Endresultate, sondern auch die thatsächlich beobachteten Werthe; so besteht auch die Möglichkeit einer Controle.

Wir schreiten nunmehr zur speciellen Mittheilung unserer Versuchsreihen. Dieselben umfasseu nur einen beschränkten Theil des hier der Forschung dargebotenen Stoffes. Sie lassen sich in zwei Hauptabschnitte einordnen, deren jeder in mehrere Versuchsreihen sich gliedert. Im ersten Abschnitt sind einige Beziehungen der Säurebildung des gereizten Muskels zur Reizgrösse dargelegt; der zweite behandelt die Abbängigkeit der Säurebildung von mechanischen Bedingungen. Auch diese Fragen sind aber keineswegs erschöpft, sondern lassen noch eine erhebliche Erweiterung: und Vertiefung der. Untersuchung za. Die vorliegende Arbeit trägt also einen durchaus fragmentarischen Charakter, was sich allerdings genugsam durch die mannichfachen Schwierigkeiten unserer Untersuchung erklärt. 


\section{I. Ueber einige Beziehungen der Sänrebildung des gereizten Muskels zur Reizgrösse.}

A. Subminimale Reizung.

Wir stellten uns die Aufgabe, zu untersuchen, ob eine elekfrische Reizung von so geringer Intensität, dass sie keinerlei sichtbare Contraction des Muskels oder auch nur einzelner Muskelfasern hervorzubringen im Stande sei, nicht doch eine nachweisbare Säuerung des Muskels zu bewirken vermöge. Selbstverständlich durfte ein etwaiges positives Resultat nur bei sehr langdanernder und sehr frequenter subminimaler Reizung erwartet werden.

Die Versuchsanordnung gestaltete sich hiernach folgendermassen: Die beiden Gastrocnemii eines durch Decapitation und Zerstörung des Rückenmarks getödteten Frosches werden sammt ihren Nerven schonend präparirt. Jedes Präparat wird mit seinem oberen Knochenende in einer Klemmschraube befestigt und mässig. belastet; die Belastung beider Muskeln war stets die gleiche. Jeder Nerv wird auf ein Paar von Häkchenelektroden gelegt. Muskelklemmen und Elektroden sind an einer Korkscheibe befestigt, die als Deckel auf einen theilweise mit. Wasser gefullten Glaseylinder aufgesetzt wird, so dass die Präparate in der feuchten Kammer hängen. Zur Erhaltung einer beliebig hohen, gleichmässigen Temperatur in derselben wird der Glascylinder in ein grosses, mit Wasser gefülltes gläsernes Gefäss gestellt; die Temperatur des Wasserbades wird durch Zugiessen von warmem und kaltem Wasser regulirt. Die Ablesung der Temperatur erfolgt an einem in die feuchte Kammer hineinragenden Thermometer, dessen Gefäss sich zwischen beiden Muskeln, von jedem kaum $1 \mathrm{~cm}$ entfernt, befand, und demnach in seinen Angaben hinreichend genau mit der Temperatur der Muskeln übereinstimmte. Den elektrischen Reiz lieferte die secundäre Rolle eines $\mathrm{Du}$ B o is - $\mathrm{R}$ e y m ond'schen Schlitteninductoriums, dessen primären Kreis der Strom eines mittelgrossen Daniell'schen Elementes durchfloss. Mit Hitlfe einer Pohl'schen Wippe mit ausgebobenem Kreuz konnte nun der Inductionsstrom bald dem einen, bald dem anderen Elektrodenpaar mit zugehörigem Nervmuskelpräparat zugeführt werden. Der Zweck dieser Anordnung erhellt aus Folgendem. Am Beginn jedes Versuches gilt es nämlich, zunächst den Minimalreiz zu er- 
mitteln; denn eine merkliche Wirkung lässt sich natürlich nur bei solchen Reizen erwarten, die nicht sehr weit unter dem Minimum liegen. Die Bestimmung des letzteren bewirkt aber stets einige, wenn auch kleine Zuckungen; auf diese könnte man dann aber eine etwaige Sänerung des subminimal gereizten Muskels zurüekführen, wodurch die Beweiskraft unserer Versuche völlig illusorisch würde. Um diesem Einwand zuvorzukommen, muss man auch den ruhenden Controlmuskel gleich oft und gleich stark zucken lassen, wie es der Versuchsmuskel that; und dies ist um so nöthiger, als die Bestimmung des Minimums wegen der noch zu besprechenden eigenthümlichen Schwankungen desselben mehrmals wiederholt werden muss. Der Verlanf eines Versuches ist also folgender: Zuerst wird durch langsames Vorrücken der secundären Rolle des Inductoriums das Minimum für das eine Präparat mittelst Einzelreiz ermittelt, hierauf die Rolle um $5 \mathrm{~cm}$ zurückgeschoben und die mechanische Unwirksamkeit des Einzelreizes und des tetanisirenden Stromes festgestellt; eventuell wird bei leichtem Flimmern des Muskels der Reiz noch mehr abgeschwächt. Dann wird die Pohl'sche Wippe umgelegt und bei wiederum vorgeschobener Rolle dès Inductoriums der Controlmuskel gereizt; bei Ungleichheit der Zuckungen beider Muskeln, wie sie ja in Folge von Erregbarkeitsdifferenzen oft vorkommt, liessen wir den Controlmuskel lieber etwas stärker oder häufiger zucken, wodurch dann der Versuch a fortiori beweisend wird. Ist hierdurch die Gleichheit beider Präparate wieder hergestellt, so wird die Wippe in ihre alte Lage gebracht und der Versuchsmuskel bei dem vorhin ausprobirten Rollenabstand „subminimal tetanisirt". Während der ganzen Versuchsdaner muss sorgfältig darauf geachtet werden, dass der Muskel nicht zuckt oder auch nur flimmert; die ersten Spuren hiervon, die noch ohne Längenänderung vor sich gehen und demnach weder durch einen Zeichenhebel nachweisbar, noch auch durch einen selbstregulirenden Apparat automatisch auszuschalten wären, entdeckt man an den obersten, dem Nerveneintritt benachbarten Fasern; tritt Flimmern ein, so wird der Strom sofort unterbrochen, der Controlmuskel compensatorisch leicht gereizt und darauf der Versuchsmuskel mit geringerer Stromesintensität weiter subminimal tetanisirt. War aber, in Folge einer augenblicklichen Unachtsamkeit unsererseits, das erste Flimmern nicht bemerkt und eine stärkere tetanische Contraction erfolgt, so wurde der Versuch so- 
fort als unbrauchbar abgebrochen. Ausser durch stetige sorgfältige Beobachtung kann man sich vor diesen unangenehmen Zwischenfällen noch dadurch schützen, dass man zeitweise das Minimum controlirt. Die Erregbarkeit des Präparates steigt nämlich häufig in den ersten 30 Minuten der Versuchsdaner oder auch noch länger, so dass die vorher subminimalen Reizwerthe minimal und mechanisch wirksam werden; in späteren Stadien der Versucbsdauer sinkt allmählich die Erregbarkeit, so dass es nöthig wird, die subminimale Reizstärke allmählich zu steigern. Wir nahmen daher die Controle des Minimums von je $20 \mathrm{za} 20$ Minuten vor, und zwar in genau derselben Weise, wie bei Beginn des Versuches, mit compensatorischer Reizung des Controlmuskels. Die ganze Versuchsdauer belief sich auf $1-1 \frac{1}{2}$ Stunden. Diese lange Versuchsdauer und die fortwährend angestrengte Aufmerksamkeit gestalten diese Beobachtungen sehr zeitraubend und mühsam, abgesehen davon, dass durch unangenehme Zwischenfälle viele Versuche unbrauchbar werden. Winterfrösche sind zu diesen Untersuchungen, wie wir uns im März 1893 iiberzengten, ganz ungeeignet; und auch die in demselben Monat an frisch eingebrachten, aber kleinen Temporarien angestellten Versuche ergaben nur theilweise ein positives Resultat. Dagegen zeigte eine im April 1893 an grossen, frisch eingefangenen Esculenten ausgefuhrte Versuchsreihe ein durchans positives, ausnahmslos eintretendes Resultat, welches wir in $\mathrm{T}$ abell e I (im Anhang) mittheilen. Die Tabelle ergibt ebenso wie die grosse Zahl unserer übrigen, bier nicht veröffentlichten, gleichartigen Versuche, den Satz, dass der subminimal gereizte Muskel, mit dem ungereizten verglichen, stets sauer reagirt. Die Säuerung ist am grössten bei ungefähr $20^{\circ}$ und wird bei höheren und niedrigeren Temperaturgraden geringer. Letzteres ist ohne weiteres aus der bekannten Herabsetzung des Stoffumsatzes in der Kälte verständlich; die kleinere Säuerungsdifferenz bei boher Temperatur dagegen ist wahrscheinlich nur als relativ kleiner aufzufassen, indem beide Muskeln durch die Wärme, wie noch neuerdings M eyerhold (13) nachgewiesen hat, eine starke Beschleunigung ihres Stoffumsatzes erfahren, dern gegenüber der geringe Zawachs durch die subminimale Reizung nicht aufkommen kann. Auffallend bleibt es iibrigens immerhin, dass das Maximum der Säurebildung bei subminimaler Reizung annähernd mit dem von Gad und Heyman's (14a) beobachteten Minimum 
der Zuckungshöhe zusammenfällt. Die Grösse der Säurebildung in unseren Versuchen entspricht, wie wir uns durch besondere, in denselben Tagen angestellte Controlbestimmungen überzeugten, etwa der Säuerung durch 10-20 Maximalzuckungen.

Das wesentlicbe Interesse dieser Versuche liegt jedoch nicht in diesen quantitativen Bestimmungen, sondern in der einfachen Constatirung der Thatsache, das s e in Reiz im M u skel eine deutliche Steigerung des Stoffumsatzeshervorrufenkann, ohne irgend welehe meehanische Wirkung a uszulösen; und zwar ist dies, soweit wir wissen, nächst Danilewsky's (15) weiter unten zu erwähnenden, nicht ganz einwandsfreien Versuchen, das erste Mal, dass es gelingt, am ausgeschnittenen, überlebenden Muskel eine solche Steigerung des Stoffumsatzes ohne mechanische Leistung direkt experimentell hervorzurufen. Dass freilich unter gewissen Umständen sich etwas derartiges fände, war schon längst bekannt; aber es war nicht versucht worden, die Wirkung dieser sogleich $z \mathfrak{u}$ besprechenden Factoren experimentell nachzuahmen. So zeigten $M$ unk (16), v. Eiselsberg (17), v. Gendre (18), A ust (19) und Gross (6) eine Beschleunigung der Todtenstarre durch den Einfluss des absterbenden Nervensystems. Vor allem aber wurde erwiesen, dass im normalen, lebendigen Organismus selbst ohne jeden Eingriff derartige chemisch wirksame subminimale Reize dem Muskel, vielleicht fortwährend, zugehen. So fanden $\mathrm{R}$ öh rig and $\mathrm{Zuntz}(20)$, Pflüger (21), Colasanti (22), Jolyet (23) eine Herabsetzung des respiratorischen Gaswechsels, Riegel (24) eine Herabsetzung der Körpertemperatur bei curaresirten Thieren; dasselbe bestätigten Pflïger (21), Adamkiewicz (25) und Erler (26) an gelähmten Thieren, Voit (27) an einem gelähmten Menschen; alles dieses sprach für eine, unter normalen Verhältnissen beständig stattfindende subminimale Innervation, einen ",chemischen Tonus"; so zeigten andererseits Cl. Beruard (28), M'Donnel (29), Ogle (30), Chandelon (31), Boehm und Hoffmann (32), Manché. (33) eine Zunabme ron Glycogen im gelähmten Muskel, dem sie eine ganz analoge Deutung gaben; so wiesen endlich Li eberme is te r (34), Röhrig und Z Zntz (20), P flüger (21) durch ihre Untersuchungen iiber die Steigerung des Gaswechsels bei Kältereizen, Paalzow (35) analog bei schmerzhaften Hautreizen, Mol esc h ot t(36), Chasanowitz (37), Pott (38) und Platen (39) .in gleicher 
Weise bei Lichtreizen, die reflektorische Natur dieses ,chemischen Tonus", seine Vermittlung dureh Reizung sensibler Nerven, nach. Endlich war von neuropatbologiseher Seite, z. B. von A d a mkiewicz (40) und Cramer (4l), allerdings theilweise mit Repristinirung der Brondgeest'schen Anschauungen, die Existenz einer normalen continuirlichen tonisehen Erregung der Muskeln betont worden. Auch möchten wir an dieser Stelle auf den allbekannten "trophischen Einfluss" des Rïckenmarks auf die Muskeln hinweisen, der unseres Wissens bisher noch keine zulängliche Deutung erhalten hat; hier haben wir eine beständige chemischo Wirkung der Innervation ohne gleichzeitigen mechanischen Effekt; sollte nicht dieser "trophische" Einfluss identisch sein mit einer beständigen subminimalen Innervation, mit dem "ehemischen Reflextonus"? Die Ansicht, dass der Muskel sein normales Fortbestehen einer beständigen Anregung seines Stoffumsatzes, also einẹm gewissen Grade der Thätigkeit verdanke, erbält anch durch die sonstigen nahen Beziehungen zwischen Thätigkeit und Ernährungszustand des Maskels, durch die Thatsachen der Arbeitshypertrophie und Inactivitätsatrophie, eine wesentliche Stütze. Ferner würde unsere Auffassung erklären, warum die degenerative Atrophie der Muskeln nur nach ihrer Abtrennung von den Vorderhörnern, nicht aber nach Läsion der Pyramidenbahn, trotz der gleichzeitigen völligen Lähmung, entsteht; nur im ersteren Falle wird ja der Reflexbogen des, ,chemischen Tonus", welcher die trophische Innervation bedingt, zerstört.

Dieselbe permanente leise Innervation, die wir soeben mit dem trophischen Einfluss des Rickenmarks identificirten, hat auch wahrscheinlich, wie bereits frühere Autoren betonten, die Aufgabe der Wärmeregulation im Organismus; ist doch nach F i ck (42) das Muskelgewebe die Hauptstätte der thierischen Verbrennungsprocesse. Mit Riicksicht hierauf ist es interessant, dass Da n i lewsky (15) bei subminimaler Reizung des Muskels eine deutliche Wärmeentwicklung beobachtete, die er in einer ganz analogen Weise, wie wir unsere Versuche, interpretirte; nur sind leider alle seine Versuche an ermüdeten, „fast nnerregbaren" Präparaten angestellt, weshalb ihre Wiederholung an frischen Muskeln sehr winschenswerth erscheint. Auch fordert die Beobachtung von Anreps (43), dass die Elasticität des Muskels nach Durchschneidung seines Nerven abnebme und unvollkommen werde, zu 
einer direkten Prüfung des Einflusses subminimaler Reizung auf die mechanischen Eigensehaften des Muskels auf. Doch liegen diese Untersuchungen ausserhalb des Rahmens unserer gegenwärrtigen Arbeit.

B. Supramaximale Reizung.

Es ist bekannt, dass der durch einzelne Inductionsscbläge gereizte Muskel bei einer gewissen Reizstärke ein Maximum seiner Hubhöhe erreicht, das auch bei weiterer Verstärkung des einzelnen Reizes nicht iuberschritten wird ${ }^{1}$ ), trotzdem aber nicht etwa das absolute, durch innere Widerstände bedingte Maximum seiner Verkürzungsfähigkeit darstellt, da es im Tefanus und in der Starre überschritten wird. Mit Recht ist diese Erscheinung von Fick (42) als eine der wunderbarsten in der Muskelphysik bezeichnet worden; in der That fragt man sich unwillkürlich, ob denn die Steigerung des Einzelreizes über das Maximum hinaus thatsächlich ganz wirkungslos bleibe oder ob sie nicht viehnehr innere Processe auslöse, die ohne äussere Formänderung einhergehen. Eine solche Frage, die für die Theorie des Zuckungsmaximums und in Folge dessen auch für unsere allgemeinen Anschauungen über die Mechanik der Muskeltbätigkeit von hervorragender Bedeutung ist, erscheint nan um so mehr berechtigt, als die Säurebildung im subminimal tetanisirten Muskel zeigte, dass der chemische Stoffumsatz keineswegs parallel mit der Grösse der mechanischen Leistung geht. Es wäre also denkbar, dass der supramaximal gereizte Muskel ceteris paribus eine grössere Säuremenge lieferte als der maximal gereizte; wir unterzogen daher diese Frage der experimentellen Prüfung. Freilich liess die Beobachtung Heidenhain's (3), dass die Wärmebildung des thätigen Muskels bei Steigerung des Reizes über das Maximum binaus nicht wächst, von vornherein keine grosse Hoffnung auf ein positives Ergebniss zu.

Die Versuchsanordnung war nun folgende. Der zum Versuch vorbereitete Gastrocnemius hing, vor Austrocknung geschützt, unter der Glocke des Baltzar'schen Myographions, auf dessen rotirende

1) Von dem durch Fick (44), La mansky (45), A. B. Meyer (46) viel discrtirten Phänomen der "übermaximalen Zuckungen" sehen wir hier $\mathrm{ab}$, da seine Bedeutung umstritten ist und auch an der Frage des Maximums principiell nichts ändern würde. 
Trommel er seine Zuckungshöhen aufschrieb. Die elektrischen Reize warden von der secundïren Spirale eines Du Bois-Reymond'schen Schlitteninductoriums geliefert, in dessen primären Kreis fünf mittelgrosse $\mathrm{D}$ a $\mathrm{n}$ i ell'sche Elemente eingeschaltet waren. Die Schliessung und Oeffnung des primären Kreises geschah rhythmisch durch den Platinspitzen-Quecksilber-Contact des Engelmann'schen (47) rhythmischen Polyrheotoms, durch dessen „gemeinschaftliche Strecke“ der primäre und secundäre Strom des Inductoriums gingen. Die Scheibe des rhythmischen Polyrheotoms wurde durch den Helmholtz'schen (48) Motor mit Centrifugal-Regulator gedreht, dessen Triebkraft zwei grosse B u n se n'sche Elemente lieferten. Da beim Abblenden eines Inductionsschlages am rhythmischen Polyrheotom der secundäre Kreis offen bleibt, was sich dann bei den in unsern Versucben zu benutzenden bedeutenden Stromstärken in sebr störender Weise durch unipolare Reizwirkungen zu erkennen gibt, so liessen wir beide Schläge durch das Präparat gehen, die wegen ihres sehr kurzen Intervalls niemals Summationswirkungen ergaben. Auf diese Weise erhielten wir sehr regelmässige Zuckungsreihen. Das Intervall zwischen je zwei Zuckungen betrug etwa 3 Sekunden. Die Reize wurden dem. Muskel zur Vermeidung einer etwaigen Säuerung durch ,innere Polarisation“ (49) indirekt, durch Vermittlung des Nerven, und zwar mittelst unpolarisirbarer Elektroden, zugeführt. Der eine Muskel wurde maximal, der andere (desselben Frosches) supramaximal gereizt. Bei unserm Inductorium wurde der Maximalreiz in der Regel bei einem Rollenabstand von ungefähr $25 \mathrm{~cm}$ erreicht; behufs supramaximaler Reizung verringerten wir denselben auf etwa $12 \mathrm{~cm}$, was mit Berücksichtigung des unverhältnissmässig raschen Wachstbums der Inductionsströme beim Annäbern der Rollen ( $46 \mathrm{~b}$ ) eine ganz enorme Differenz der maximalen und supramaximalen Reizstärke gibt. Uebrigens ändern sich die Versuchsresultate nicht wesentlich, wenn man den supramaximalen Reiz bei völlig übereiuander geschobenen Rollen wählt; nur werden sie etwas unregelmässiger. T a belle II gibt eine Uebersicht der Versuchsresultate.

Aus den Zahlen dieser Tabelle ergibt sich nun in voller Uebereinstimmung mit dem oben angefuhrten Satze Heidenhain's (3), dass dersupramaximal gereizteMuske]

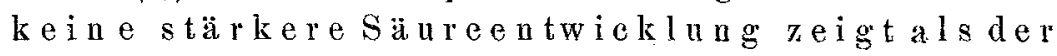


maximal gereizte. Entweder ist also die supramaximale Steigerung des Reizes völlig wirkungslos, oder die durch dieselbe noch weiter gesteigerten ehemischen Umsetzungen gehen obne Säurebildung und Wärmeentwicklung einher. Betrachten wir von diesem Gesichtspunkt aus die Deutang, welche Kohnstamm (50) dem Zuckungsmaximum gegeben hat! Er erklärt dasselbe, rom Standpunkte der Fick-Gad-Heymans'schen (51) Theorie, durch eine verstärkte Interferenz der beiden mit dem Reiz noch weiter wachsenden antagonistischen Processe, welche die positive bezw. die negative Zustandsänderung bedingen, indem der zweite, der Erschlaffungsprocess, mit wachsender Reizgrösse eine stärkere Steigerwng ertahrt als der erste. Man wird es nun schon schwer begreiflich finden, wie bei supramaximalem Reiz der erste Process, der doch sicher positive chemische Arbeit leistet, noch weiter zunehmen kann, ohne dass dies weder chemisch noch thermisch nachweisbar ist. Noch mehr aber müssen wir gegen die Deutung. des zweiten Processes Einspruch erheben, den Autor ebenfalls ausdruicklich als positive clemische Arbeitsleistung auffasst und von dessen Verbrennungswärme er sogar spricht; denn dann müsste doch sicherlich eine grössere Wärmeentwicklung des supramaximal gereizten Muskels nachweisbar sein, was die Versuche H e i d e n h a in's streng verneinen; die Säurebestimmung könnte allerdings bier im Stiche lassen, da ja der zweite Process in einer Weiterverbrennung des durch den ersten gebildeten Zwischenproduktes, also etwa der Milchsäure, besteben soll. Will man also die angegebene Deutung des Zuckungsmaximums nach der Fick-Gad-H e y mans'schen Theorie festhalten, so muss man jedenfalls die soeben dargelegte Auffassung des Erschlaffungsprocesses fallen lassen. Wäre es nicht vielleicht richtiger, den zweiten Process, statt ihn das Zerstörungswerk des ersten nur fortsetzen zu lassen, vielmehr als echten Restitutionsprocess, als einen syntbetischen Vorgang mit negativer cherniseber Arbeit aufzufassen, so dass beide Processe ungefähr in demselben gegensätzlichen Verhältniss zu einander ständen, wie Hering's Dissimilation und Assimilation? Auf diese Weise entginge man dem obigen Widerspruch; doch wagen wir nicht, diese Ansicht vorlänfig für mehr als eine Vermuthung auszugeben. 
Beiträge zur Kenntniss der Säurebildung und des Stoffumsatzes etc. 371

\section{Ueber die Abhängigkeit der Säurebildung des Muskels von mechanischen Einwirknngen.}

A. Versuchsplan und Literaturübersicht.

Jede Untersuchung über die Abhängigkeit der Vorgänge im Muskel von mechanischen Einwirkungen muss von der grundlegenlen Beoabachtung $\mathrm{H}$ e i d e $\mathrm{n}$ h a i $\mathrm{s}$ (3) ausgehen, dass die Leistung des Muskels unabhängig vom Reiz mit wachsender Spanlung zunimmt. Seitdem haben viele Autoren, insbesondere F i c k (52), v. Kries (53), Schenck (54), Sogalla (55), Santesson (56) die Thatsache einer weitgehenden Abhängigkeit der Muskelprocesse von den mechanischen Bedingungen der Muskelthätigkeit bestätigt. Das Wesen dieses Abhängigkeitsverhältnisses ist aber bisher völlig unbekannt. Zwar hatte auch in dieser Beziehung $\mathrm{H}$ e i d e $\mathbf{n}$ h a in (3) einen Schritt gethan, indem er nachwies, dass die Abhängigkeit des Stoffumsatzes im Muskel von der Spanuung nicht auf den Moment des Reizes beschränkt ist, sondern während der ganzen Dauer der Zuckung in unveränderter Gültigkeit fortbesteht; und analog war durch die Versuche von L a n d a $u$ und Pacully (4) sowie von Steiner (57) die Gültigkeit des Gesetzes während des Erschlaffungsstadiums nachgewiesen. Immer war es jedoch auch bei diesen Versuchen ein $\mathrm{t}$ h ä $\mathrm{t} \mathrm{i} g \mathrm{er}$, gereizter Muskel, an welchem experimentirt warde; und so musste die Frage, ob die Wirkung mechanischer Bedingungen anf den Stoffumsatz nur unter Einfluss der Reizung oder ganz unabhängig von dieser, durch eine direkte Einwirkung auf den Muskel zu Stande kommen, eine offene bleiben. Es erschien daher geboten, den Einfluss mechanischer Bedingungen auf den ungereizten, unthätigen, d. h. keine mechanische Leistung vollführenden Muskel zu prüfen; sollte sich etwa a ch hier eine Steigerung des Stoffumsatzes ergeben? Dies ist die Aufgabe, die wir uns gestellt hatten und an der Hand von Säurebestimmungen zu lösen versuchten. Ehe wir jedoch zur Mittheilung unserer Resultate übergehen, erscheint es angemessen, die Erfahrungen, welche frühere Autoren iber den Einfluss mechanischer Bedingungen auf den ruhenden Muskel gesammelt haben, soweit sie für nuser Thema von Interesse sind, zu uiberblicken.

E. Pflüger, Archiv f. Physiologie. Bd. 56. 
Schon das eigenthiimliche Verhalten der Dehnungscurve machte manche Autoren stutrig und liess sie darin den Effekt complieirter chemischerProcesse sehen, welche durch dic Delinung hervorgerufen seien; diese Ansicht sprachen Montgomery (58) und v. Kries (59) aus, und auch wir konnten uns; gelegentlich einer früheren Untersuchung, derselben nur anschliessen (60).

Ob eine plötzliche Dehnung des Muskels diesen zu einer Verkürzung reize, ist nach den Angaben verschiedener Autoren als unentschieden zu erachten; wäbrend z. B. Rood (61) einen solchen Einfluss behauptet, wird er von anderen, z. B. E. F ick (62) ausdriicklich geleugnet.

Ebenso unsicher ist die Frage, ob der ruhende Muskel bei seiner Dehnung Wärme entwickelt. Allerdings erhielten Me y erste in und Thiry (63), He iden ha in (64), We s terman (65), S chmulewits ch (66), B lix (67), Danilewsky (68) bei derartigen Versuchen positive Resultate; jedoch führt Heidenhain dieselben auf Fehlerquellen zurïck, und Westermann und Danilewsky stellten dieselbe Erscheinung auch am todten Muskel fest; iberdies zeigte Danilewsky, dass die durch passive Erschütterung eines lebenden Muskels gesetzte Erwärmung meist kleiner ist als dem Aequivalent der Fallarbeit entspricht und demgemäss vollständig auf rein pbysikalischemWege erklärt werden kann.

Noch misslicher stebt es um die Frage, ob die elektromotorischen Eigenschaften des ruhenden Muskels durch mechanische Einwirkungen eine Aenderung erfahren, zumal hier die Untersuchung durch Aenderungen der Neigungsströme ersehwert ist. Doch hat D a B o is - R e y m ond (69) bei Dehnung regelmässiger Muskeln oft eine negative Schwankung gesehen; dasselbe bestätitigen neuerdings v. Korányi und Vas (70).

Ein ermüdender Einfluss der blossen Spannung wird von Kronecker (71), Harless (72) und Leber (73) einstimmig geleugnet; die letzteren beiden Autoren verwendeten allerdings nur kleinere Gewichte und geringe Einwirkungszeiten. Dagegen ist ein sicherer positiver Einfluss der Spannung auf die Erstarrung nachgewiesen; E. Krause (74) und Wundt (75) beobachteten, dass die Extensoren fruher erstarren als die Flexoren; E. Krause ging so weit, dass er die Spannung als nothwendige Bedingung für das Zustandekommen der Erstarrung betrachtete, während W andt zeigte, dass auch der ungespannte Muskel, freilich lang- 
samer, erstarrt. Nasse (76) bestätigte diese Resultate. - Eine Untersuchung ïber den Einfluss mechaniseher Bedingungen auf die chemische Beschaffenheit des Muskels, speciell auf seine Säurebildung, existirt unseres Wissens bisher nicht.

B. Versuche über den Einfluss der constanten Spannung auf die Säuerung des Muskels.

Zur Beschreibung der Versuchsanordnung genügen wenige Worte. Die beiden Gastrocnemii eines Frosehes wurden mit kurz abgeschnittenen Nerven in die früher beschriebene feuchte Kammer (S. 363) gehängt und diese entweder in den Eisschrank oder anf den Brïtofen gestellt. Der eine Muskel war unbelastet; der andere trug ein Gewicht, dessen Grösse in verschiedenen Versuchen verschieden war und innerhalb $100 \mathrm{gr}$ bis $1000 \mathrm{gr}$ variirte. Letztere Belastung ist ungefähr die äusserste, die man der Zugfestigkeit des Gastrocnemins zumuthen kann, da in mehreren dieser Versuche der Muskel zerriss. Die Einwirkungszeit der Belastung betrug stets drei Stunden. Dann wurde die Säurebestimmung gemacht. In $\mathrm{T}$ a b e ll e III finden sich die Resultate dieser Versuche zusammengestellt.

Das ibereinstimmende Ergebniss aller Versuche ist nun eine merkliche Säuerung des belasteten Muskels gegenüber dem un belastete n, die mit steigender Temperatur und steigender Belastung bis zu sehr erheblichen Werthen zunimmt. Die bei maximaler Belastung und einer Temperatur von $25^{0}$ beobachtete Säuremenge erreicht fast dieselbe Grösse, wie bei einer Zuckungsreihe von 200 Maximalzuckungen; rgl. Tab. II.

Die Tbatsache, dass die blosse Spannung den Stoffumsatz eines Muskels vermehrt, lässt uns manche bisher räthselbafte klinische Erscheinungen verstehen. Wir denken hierbei an die bedeutende Atrophie, der ein abnorm entspannter Muskel, z. B. der Extensor cruris quadriceps nach Querfractur der Patella, binnen kurzer Zeit verfällt und die keinesfalls auf blosse Inactivität zurückzuführen ist. Nun aber, da wir wissen, dass die Belastung auch im ruhenden Muskel eine Steigerung des Stoffumsatzes bewirkt, lässt sich die "Entspannungsatrophi e" auf den Fortfall dieser Anregung, anf eine Herabsetzung des normalen Stoffwechsels zurickfubren. Fs tritt also diese Erscheinung in voll- 
ständige Analogie zu der Arbeitshypertrophie des Herzens bei Klappenfehlern, Nepbritis ete., zu der Hypertrophie der Blasenmuskulatur bei Harnröhrenverengerung, Thatsachen, welche durch Heidenhains (3) Entdeckung der Abbängigkeit des Stoffumsatzes im arbeitenden Muskel von seiner Spannung ins rechte Licht gestellt worden sind; unsere Versuche zeigen, dass dieser Satz auch für den rubenden Muskel gültig ist. Man könnte aus unseren experimentellen Erfahrungen vielleicht sogar bemerkenswerthe Anweisungen für die Therapie solcher "Entspannungsatrophieen" ziehen; es wird naturlich vor allem darauf ankommen, die normale Spannung des Muskels wieder herzustellen, sei es durch exakte Vereinigung der Patella, sei es, wie z. B. bei subcutanen Muskelzerrẹissungen, durch die Muskelnaht. Ans demselben Gesichtspunkte ist vielleicht auch der therapeutische Werth der Tenotomien bei Strabismus zu betrachten.

Die beständige Spannung, unter der alle normaien Muskeln in Folge der anatomischen Anordnung ihrer Insertionen stehen, dieser sog. "mechanische Tonus" hat also nicht bloss, wie bisher geglaubt wurde, eine Verfeinerung der Coordination und Ersparniss an Muskelkraft zur Folge, sondern dient ausserdem noch zur wirksamen Erhöhung des „chemischen Tonus“.

C. Versuche ïber den Einfluss rhythmischer Belastung und Entlastung auf die Säuerung des Muskels.

Wenn schon die constante Spannung den Stoffumsatz des Muskels erheblich zu steigern vermochte, so musste dies in noch höherem Grade bei wechselnder Spannung und Entspannung der Fall sein, da hierbei die mechanischen Verhältnisse, wie sie beim arbeitenden belasteten Muskel obwalten, möglichst vollkommen nachgeahmt werden.

Zur rhythmisch wechselnden Belastung und Entlastung diente uns folgender Apparat. Der Gastrocnemius hing in einem, oben und unten offenen, mit feuchtem Fliesspapier austapezirten Glascylinder. Sein unteres Ende war durch einen Faden mit dem einen Ende eines leicht drehbaren eisernen Wagebalkens verbunden; an demselben Ende hing die Last. Das andere Ende des Wagebalkens wurde nun durch elektromagnetische Anziehung eines Ankers herabgedriickt und so der Muskel entlastet; bei Unter- 
brechung des magnetisirenden Stromes fiel das vorher gehobene Gewicht natürlich wieder herab und spannte den Muskel. Schliessung und Oeffnung des Stromkreises für den Elektromagneten geschah rhythmisch, und zwar mit beliebig regulirbarer Frequenz, durch ein Mälzel'sches Metronom. Der Controlmuskel hing während der Versuchsdauer mit dem gleichen Gewicht constant, wie der andere rhythmisch, belastet, in der feuchten Kanmer. Die Versuchsdauer betrug stets eine Stunde, die Belastnng bei beiden Muskeln je $50 \mathrm{gr}$, die Temperatur ungefähr $20^{\circ}$. Die Frequenz des Belastungswechsels war in der ersten Versuchsreihe 25, in der zweiten 100 pro Minute. T a b e lle IV giebt eine Uebersicht der Resultate dieser Versuche.

Die Tabelle beweist, dass rhythmisch wechselnde Spannung und Entspannung den Stoffumsatz des Muskels stärker anregt als constante Spannung, die bei so kleinem Gewicht and der. verbältnissmässig kurzen Einwirkungszeit von einer Stunde fast wirkungslos geblieben wäre; die Säuerung ist um so stärker, je grösser die Belastung und je frequenter der Belastungswechsel. Jedoch nimmt die Säurebildung mit zunelmender Zahl der Erschïtterungen nur nach absolutem Masse zu, nach relativem Masse dagegen ab; sie wächst also unverhältnissmässig langsamer als die an deın Muskel geleistete Fallarbeit; wir gehen wohl nicht fehl, wenn wir dies als ein Zeichen der Ermüdung des Muskels auffassen; die späteren Erschiitterungen vermögen den, bereits im Anfang des Versuches stark in Anspruch genommenen Stoffumsatz nicht mehr in gleichem Grade anzuregen wie die ersten.

Anch die Versuche dieses Kapitels sind für die klinische Medicin von Wichtigkeit; sie machen den günstigen Effekt passiver Bewegungen auf die Muskulatur bis zu einem gewissen Grade verständlich.

Die in den beiden letzten Kapiteln mitgetheilten Versuchs. reiben lehren, dass auch der ruhende Muskel durch mechanische Einwirkungen eine Erhöhung seines Stoffumsatzes erfahren kann. Nun taucht sofort die Frage auf: Ist etwa die Steigerung des chemischen Umsatzes, welche der thätige, mechanische Arbeit leistende Muskel durch ver- 
mehrte Spannung gegenüber dem unbelasteten erfahrt, bloss bedingt durch einfache Addition der Wirkungen beider, unabbängig neben einander bęstehenden Faktoren, Reiz und Belastung, oder findet eine gegenseitige B eeinflussung, also eine functionelle Verknüpfung beider Faktoren statt? Zur Beantwortung dieser wichtigen Frage ist es erforderlich, zu erforschen, ob dieselbe mechanische Einwirkung eine grössere oder nur eine ebenso grosse Säuremenge im thätigen Muskel hervorbringt wie im rubenden. Ist ersteres der Fall, so ist die obige Alternative im Sinne einer functionellen Verknüpfung beider umsatzsteigernder Momente, Reiz und Belastung, entschieden; trifft dagegen letzteres $z \mathfrak{u}$, so besteht nur eine additionelle Verknüpfung. Diese experimentelle Untersuchung soll uns sogleich eingehend beschäftigen.

D. Vergleich der Wirkungen mechanischer Momente a uf den Stoffumsatz des rubenden und des thätigen Muskels.

Durch die frither erwähnten Versuche von Heidenhain (3), Landau und Pacully (4), sowie Steiner (57) ist erwiesen, dass gewaltsame Dehnung des Muskels während des Erschlaffungsstadiums der Zuckung den Stoffumsatz steigert. Eine quantitative Bestimmung der gebildeten Säuremenge war damals nicht gemacht worden, nunmehr aber mit Hilfe der verfeinerten Methode sehr wohl möglich. Vergleichen wir nun ceteris paribus die Säuremenge, welche in derartigen Versuchen durch die mechanische Einwirkung hervorgebracht wird, mit derjenigen, welche die wechselnde Spannung und Entspannung am ruhenden Muskel in der vorigen Versuchsreihe erzeugte, so haben wir die Lösung unserer Frage. Der Vergleich muss, da an einem Muskelpaare immer nur die Hälfte eines Versuches ausführbar ist, natürlich auf statistischem Wege geschehen.

Um den Einfluss der passiven Dehnung durch das fallende Gewicht während-des Erschlaffungsstadiums zu ermitteln, gilt es, den einen Muskel diesem Einfluss zu entziehen, d. h. ihn auf der Höhe der Zuckung zu entlasten. Zu diesem Zweck bedienten wir uns zuerst des Arbeitssammlers von Fick (77). Jedoch erhielten wir bei Gebrauch dieses Apparates eine nur sehr unregelmässige Bestätigung des positiven Resultates der früheren Autoren. Die 
Erklärung hierfür liegt in folgendem Umstande. Die Last, welche der Muskel am Arbeitssammler hebt, hat in Folge ihrer Verknüpfung mit den grossen Schwungmassen des Rades ein sehr bedeutendes Trägheitsmoment, so dass sie noch zu steigen fortfährt, wenn der Muskel sich bereits wieder ausdehnt. Natürlich trifft dann die herabfallende Last nicht mehr den erschlaffenden, sondern den bereits ruhenden Muskel, lässt also die im Stadium der Erschlaffung sich abspielenden activen Processe unbeeinflusst; die Forderung unseres Versuchsplanes bleibt demnach unerfüllt. Es blieb also nichts übrig, als die Verwendung des Arbeitssammlers ganz aufungeben. Hiermit soll natürlich nicht etwa die Beweiskraft der mit diesem Apparat ausgefiihrten Untersuchungen von Fick (77) and Danilewsky (68) angetastet werden. Vielmehr hat letzterer Autor auf das erhebliche Trägheitsmoment des Apparates ausdriicklich aufmerksam gemacht und dasselbe für seine und F i ck's Versuche geradezu für nothwendig erklärt, um sie vor dem bekannten Einwande Heidenhain's (4) zu schützen. Man beachte ferner, dass die Zwecke, welche Fick und Danilewsky verfolgten, von dem unsrigen durchaus verschieden sind; sie wollen prüfen, ob die durch das dehnende Gewicht im Muskel erzeugte Wärmemenge der Fallarbeit äquivalent ist, und für sie ist es daher im Grunde genommen gleichgültig, wie auch Danilewsky hervorhebt, ob das Gewicht durch die Thätigkeit des Muskels oder durch fremde Kraft gehoben wurde; ihre Frage ist also eine rein physikalische, nämlich die, ob das Princip der Erhaltung der Energie auch im lebenden, thätigen Muskel giiltig sei; wir dagegen stellen eine Frage von rein physiologischem Interesse, die Frage nämlich, ob mechanische Einwirkung die activen Processe im erschlaffenden Muskel zu steigern vermöge; was daher jene Forscher peinlichst vermieden, die passive Dehnung während der spontanen Erschlaffung, das gerade müssen wir erstreben. Daher bleiben auch die Resultate von Fick und Danilewsky, unbeschadet der Unverwendbarkeit des Arbeitssammlers für unsere Zwecke, zu Rechte fortbestehen; und wesentlich, um Missverständnissen in gedachter Richtung vorzubeugen, sind wir auf diesen Punkt näher eingegangen.

Wir kehrten also zu dem Apparate zurück, den $\mathrm{H}$ e i d e nh a i n and seine Schüler bei ihren Versuchen erprobt hatten. Betreffs der speciellen Einrichtung desselben verweisen wir auf die Abhandlungen dieser Autoren (4), insbesondere die von St einer (57), 
wo sich auch eine Abbildung des Apparates findet. Hier sei nur kurz erinnert, dass das Princip desselben in einer Zahnstangensperrung besteht, welche den Myographionhebel wohl aufwärts, nicht aber abwärts gehen lässt und demnach den Muskel auf der Höhe der Zuckung entlastet; kurz vor der nächstfolgenden Zuckung, also nach völliger spontaner Erschlaffung des Muskels, wird die Sperrung gelöst. Bei den Versuchen mit Dehnung des erschlaffendeu Muskels wird die Sperrung einfach vor Beginn des Versuches ansgeschaltet; bei dieser Anordnung verhält sich dann die Belastung wegen des sebr geringen Trägheitsmomentes fast wie eine frei fallende und dehnt daher den Muskel noch während seiner spontanen Erschlaffung. Dieser einfache Apparat erfüllt vollständig die Anfordernngen unseres Versuchsplans; den besten Beweis hierfiir liefert die befriedigende Constanz der quantitativen Ergebnisse. Die Zuckungen wurden auf die berusste Glasplatte des Pflügerscben Myographions mit doppelter Vergrösserung geschrieben; von je $20 \mathrm{zu} 20$ Zuckungen wurde die Platte mit der Hand etwas verschoben. Die Zahl der Zuckungen betrug stets 200, die Belastung $50 \mathrm{gr}$. Die Arbeit wird sogleich nach Beendigung des Versuches durch Ausmessung der Zuckungshöhen berechnet und einheitlich reducirt. Zur Reizung dienten maximale Schliessungsinductionsschläge, die dem Präparat durch das rbythmische Polyrheotom Eng elmann s (47), und zwar gemäss den Erfahrungen frïherer Autoren direkt, zugeleitet wurden... Der eine Muskel zuckte mit, der andere ohne Entlastung auf der Höhe; die stets resultirende positive Sänerungsdifferenz zu Gunsten des letzteren kann natürlich nur auf die passive Dehnung bezogen werden und wurde in allen Versuchen auf eine gemeinsame Einheit der Fallarbeit des dehnenden Gewichtes, und zwar auf 100000 grmm reducirt.

Tabelle V gibt eine Zusammenstellung der Resultate dieser Versuchsreihe.

Es ergibt sich zunächst eine vollkommene Bestätigung der Beobachtungen von Heid enhain, Landau und Pacully (4); di e Säuerungs differenz zn Gunsten deswährendseiner spontanen Erschlaffung. gedehnten Muskels beträgt $16-36 \%$ des Gesammtwerthes der Säuerung, ist also recht erheblich.

Eine weitere interessante Bestätigung dieses Ergebnisses liegt darịn, dass der auf der Höhe der Zuckung entlastete Muskel in fast allen Versuchen eine grössere Summe mechanischer Arbeit 
leistet als der während der Erschlaffung gedehnte; vergl. Tab. V; diese Differenz entfällt, wie wir aus unseren Ordinatenmessungen nachweisen konnten, stets auf die zweite Hälfte der Zuckungsreihe; der gedebnte Muskel ermüdet also schneller, was wiederum für den erhöhten Stoffumsatz spricht.

Da natürlich die am gedebnten Muskel durch das frei fallende Gewicht geleistete Arbeit gleich ist derjenigen, welche der Muskel selbst durch Hub des Gewichtes leistete, so gibt die letzte Spalte der Tabelle $V$ unmittelbar die dureh äussere mechanische Einwirkung im spontan erschlaffenden Muskel gebildete Säuremenge an, reducirt auf $1,0 \mathrm{gr}$ Muskelgewicht und $100000 \mathrm{gr}$ Fallarbeit.

Vergleichen wir nun diese Säurebildung ibrer Grösse nach mit der durch mechanische Einwirkungen am ruhenden Muskel erzeugten und auf die gleichen Einheiten bezogenen (Tab. IV) Säuremenge, so ergibt sich die erstere, am thätigen Muskel beobachtete, stets als bedeutend grösser. Diese Differenz aber muss in Wahrheit sogar noch grösser sein; denn ein Theil derselben wird durch einen principiellen, allerdings unvermeidlichen Fehler unserer Methode verdeckt, indem ja auch in den Versuchen mit Entlastung auf der Höbe dennoch nachträglich, nach völliger Erschlaffung, der Muskel durch das fallende Gewicht bei der Lösung der Sperrung mechanisch gedehnt wird und so eine gewisse Anregung seines Stoffumsatzes erfäbrt; ohne diese wiirden die Werthe in der letzten Spalte unserer Tabelle natürlich noch grösser sein. Ja, man könnte versucht sein, diese Werthe direkt als Ausdruck der Differenz zwischen der Säurebildung durch mechanische Einwirkung während der Erschlaffung und nach derselben, d. h. während der Rube anzusehen; indessen wäre dies nicht exakt, da bei der manuellen Lösung der Sperrung das Gewicht sich nicht wie ein frei fallendes verhält, sondern langsamer herabgelassen wird. Immerhin ist der hier angezogene Umstand für unser ohnedies schon positives Resultat noch sebr günstig.

Es stehtalsofest, dassdiegleichemeebanisehe Einwirkung am gereizten thätigen Muskel den Stoffumsatz weit stärkererböbt, als ceteris paribus a $m \mathrm{r} u \mathrm{~h}$ enden. Demnach kann die Umsatzsteigerung im ersten Fall nicht a uf einfacher Addition beider in diesem Sinne wirkenden Momente, Reiz und Belastung, sein, sondern es muss eine functionelle Verkn ü fung beider Faktoren bestehen, der Art, dass das Vorhandensein des einen, des Reizes, 
die Wirkung des gleichzeitig existirenden anderen, der mechanischen Verhältnisse, steigert.

Aus diesem Gesichtspunkte ergibt sich nun auch eine Erklärung für das merkwürdige Resultat von Nawalichin (78) und Dan ilewsky (15), dass die Wärmeentwicklung mit zunehmendem Reiz nicht proportional, sondern unverhältnissmässig schneller wächst; sie ist eben das Produkt nicht des Reizes allein, sondern auch der Belastung, und sie wächst daher bei Steigerung des Reizes nicht allein durch diese, sondern ausserdem noch mittelbar dadurch, dass unter dem Einfluss des stärkeren Reizes die gleiche Belastung den Úmsatz direkt stärker anregt, als vorhin. Die Möglichkeit einer Erklärung in der angedeuteten Richtung hat bereits F i c k (42) vorausgesagt.

\section{Schlussbetrachtungen.}

Mehrere der im Vorigen mitgetheilten Versuchsreihen erfordern noch eine gemeinsame Besprechung von allgemeinerem Gesichtspunkte aus, da ihre Zusammenstellung und Vergleichung zu neuen interessanten Schlüssen Anlass gibt.

Es ist längst bekannt, dass der ruhende Mnskel beständig denselben Gaswechsel zeigt wie der thätige, freilich in geringerem Masse, dass er ferner olne jeden äusseren Eingriff beständig Säure producirt, und dass, ebenso wie im thätigen, so auch im ruhenden Muskel der Stoffumsatz durch Wärme gesteigert werden kann. Alle diese Thatsachen nöthigten zu der Annahme, dass auch im ruhenden, $d . h$. keine mechanische Arbeitsleistung vollziehenden Muskel ein beständiger chemischer Umsatz stattfinde und dass dieser Stoffumsatz principiell identisch sei mit demjenigen, welcher im gereizten arbeitenden Muskel waltet. Der Satz P fl ïger's (79): „Die Schulausdriicke Ruhe und Thätigkeit des Nerven beziehen sich in Wahrheit nur auf Gradationen desselben Zustandes" ist daher mit gleichem Recht auch auf den Muskel anwendbar. Fuir diesen Satz können nun mehrere unserer Versuchsreihen als neue gewichtige Beweise eintreten. Wir fanden, dass Reiz und Belastung auch im ruhenden Muskel den chemischen Umsatz steigern, in gleicher Weise, wie sie es im thätigen, mechanische Arbeit leistenden Muskel thun. Der innere Mechanismus, durch welchen Reiz und Belastung in beiden Zuständen des Muskels den gleichen Effekt ansüben, ist also in beiden Fällen derselbe. Einerseits wird hierdurch aufs nene bewiesen, dass in beiden Zuständen 
wesentlich gleiche Vorgänge walten, die in der "Ruhe“ nur cinen geringeren Grad von Intensität erreichen als in der "Thätigkeit" des Muskels; andererseits aber erhalten die Gesetze von der umsatzsteigernden Wirkung des Reizes und der Spannung einen weit allgemeineren Charakter als sie bisher besassen.

Welche Vorstellung soll man sich num aber nach der vorgetragenen Auffassung davon machen, dass nicht jeder Werth der Intensität des chemischen Umsatzes zugleich auch eine mechanische Leistung erzeugt, sondern dass letzteres erst von einem gewissen Schwellenwerthe ab der Fall ist? Hierfür dürfte zweierlei zu beachten sein. Zunächst ist es nicht die absolute Grösse des Stoffumsatzes innerbalb einer endlichen Zeit, welche den mechanischen Effekt bestimmt, sondern der Werth desselben in einem einzelnen Zeitdifferential; bei langdauernder subminimaler Reizung oder constanter Spannung wird, wie wir uns mehrfach selbst überzeugten, mehr Säure gebildet als bei einer Reihe von Maximalzuckungen, ohne dass eine Spur mechanischer Arbeit geleistet wird; der Grund liegt darin, dass dort der Stoffumsatz sich auf sehr lange Zeit erstreckte, hier dagegen in küzester Frist erfolgte. Dass jedoch der mechanische Effekt nicht bei jedem beliebig kleinen Werthe des Unsatzes im einzelnen Zeittheilchen eintritt, sondern erst bei einem gewissen Schwellenwerth, das erklärt sich wohl durch das Trägheitsmoment der zu bewegenden Maschinentheile im Muskel, sowie ja auch der Nerv auf allzu schnelle elektrische Schwankungen nicht mebr reagirt.

Schliesslich müssen wir noch auf einen Einwand Riucksicht nehmen, der vielleicht gegen unsere Deutung des Einflusses der subminimalen Reizung und der Spannung auf den ruhenden Muskel erhoben werden könnte. Man wird vielleicht behaupten, dass die Säuerung des Muskels durch diese Momente nichts weiter bedéte als eine Beschleunigung der Starre, wie sie ja für die Spannung auch auf anderem. Wege (S. 18) direkt nachgewiesen sei. Hierauf ist zu erwidern, dass diese Erklärung nur scheinbar der unserigen entgegensteht, in Wirklichkeit aber mit ibr identisch ist ; denn da der beständige chemische Umsatz im ausgeschnittenen, ruhenden Muskel nichts Anderes ist als der allmähliche Erstarrungsprocess and stets mit der völligen Erstarruug endigt, so ist naturgemäss jede Steigeruug dieses Umsatzes gleichzeitig auch eo ipso cine Beschleunigung der Erstarrung, wie ja auch die mechanische Arbeitsleistung des zuckenden Muskels in gleichem Sinne 
wirkt. Thatsache bleibt jedenfalls, dass durch unsere Versuchsbedingungen gewisse chemische Processe im Muskel gesteigert werden; und es ist wohl nur gerechtfertigt, diesen Stoffumsatz von dem 1 e b e n d e n Zustande des Muskels abzuleiten. In diesem Sinne spricht sich auch neuerdings Salk ow ski (80) aus, indem er sagt: "Es ist mir viel wahrseheinlicher, dass der Muskel nicht Milchsäure bildet, weil er abstirbt, sondern weil er lebt und sie nur bildet, so lange er lebt." Wir möchten hinzufügen, dass der Muskel auch nurdeshalberstart, weil er lebt und nur so lange in der Erstarrung fortschreitet, als er noch lebt; $d$ as Ende der Erstarrung ist der Tod; „die Uhr ist abgela ufen" (81). Die beständige Selbstzersetzung der lebenden Muskelsubstanz, welche den Muskel, so lange er ein Glied eines sich selbständig ernährenden Organismus ist, zu Leben and Leistungen befähigt, muss mit derselben Consequenz den ausgeschnittenen, seinen Ernährungsbedingungen entzogenen Muskel verzehren und zum Tode führen; und das um so schneller, je intensiver dieser Selbstzersetzungsprocess vor sich geht; daher erfolgt die Erstarrung bei erhöhter Temperatur und beim Warmblüter viel rapider. Der isolirte Muskel verhält sich also ganz analog wie ein des Sanerstoffs beraubter Organismus, z. B. wie Pflügers (81) Frösche in reinen Stickstoff; beide tötet, so paradox es klingen mag, ibr eigenes Leben. - Ruhe, Thätigkeit und Erstarrung des Muskels sind also principiell identische Processe.

Tabelle I.

Subminimale Reizung.

Rana esculenta. Reizdauer: 1 Stunde. Belastung: $20 \mathrm{gr}$.

\begin{tabular}{|c|c|c|c|c|}
\hline Datum & 'Temperatur & $\begin{array}{c}\text { Muskel- } \\
\text { gewicht } \\
\text { gr }\end{array}$ & $\begin{array}{l}\text { Säurels } \\
\text { beob- } \\
\text { achtet }\end{array}$ & $\begin{array}{l}\text { dung in mgr } \\
\begin{array}{|l}\text { reducirt auf } \\
1,0 \text { gr Muskel- } \\
\text { gewicht }\end{array}\end{array}$ \\
\hline $\begin{array}{ccc}\text { 10. } & \text { IV. } & 1893 \\
\text { 11. IV. } & 1893 \\
" & " & " \\
" & " & "\end{array}$ & $\begin{array}{c}18,5-20,5^{0} \\
19-21^{0} \\
18-20^{0} \\
19,5-20,5^{0}\end{array}$ & $\begin{array}{l}0,9 \\
1,0 \\
0,7 \\
0,8\end{array}$ & $\begin{array}{l}2,25 \\
1,2 \\
0,5 \\
1,1\end{array}$ & $\begin{array}{l}2,5 \\
1,2 \\
0,7 \\
1,4\end{array}$ \\
\hline $\begin{array}{c}\text { 12. IV. } 1893 \\
" \# " n\end{array}$ & $\begin{array}{l}12^{\circ} \\
13,5^{\circ}\end{array}$ & $\begin{array}{l}0,7 \\
0,8\end{array}$ & $\begin{array}{l}0,21 \\
0,32\end{array}$ & $\begin{array}{l}0,3 \\
0,4\end{array}$ \\
\hline $\begin{array}{c}\text { 12. IV. } 1893 \\
" \# " ~\end{array}$ & $\begin{array}{l}29-30^{0} \\
28-30^{0}\end{array}$ & $\begin{array}{l}1,2 \\
0,9\end{array}$ & $\begin{array}{l}0,22 \\
0,36\end{array}$ & $\begin{array}{l}0,18 \\
0,4\end{array}$ \\
\hline
\end{tabular}


Beiträge zur Kenntniss der Säurebildung und des Stoffumsatzes etc.

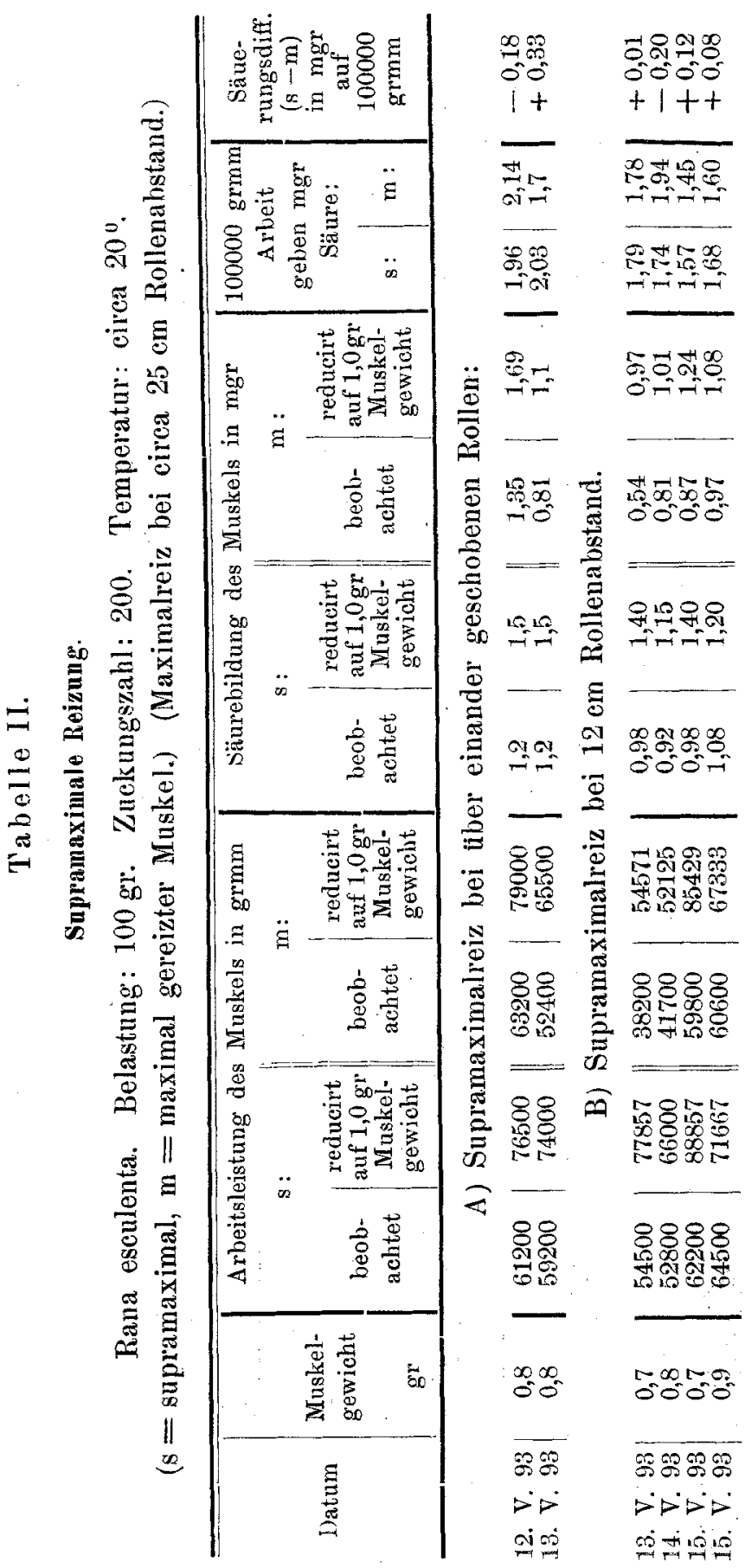




\section{Tabelle III.}

Constante Belastung.

Rana esculenta. Versuchsdauer: 3 Stunden.

\begin{tabular}{|c|c|c|c|c|c|}
\hline Datum & $\begin{array}{c}\text { Belastung } \\
\text { gr }\end{array}$ & $\begin{array}{l}\text { Muskel- } \\
\text { gewicht } \\
\text { gr }\end{array}$ & $\begin{array}{l}\text { Säureb } \\
\text { beob- } \\
\text { achtet }\end{array}$ & $\begin{array}{l}\text { lung in } \mathrm{mgr} \\
\mid \begin{array}{l}\text { reducirt auf } \\
1,0 \text { gr Muskel- } \\
\text { gewicht }\end{array}\end{array}$ & \\
\hline $\begin{array}{l}\text { 15. V. } 1893 \\
\text { 16. } " n\end{array}$ & $\begin{array}{l}100 \\
100\end{array}$ & $\begin{array}{l}0,6 \\
0,7\end{array}$ & $\begin{array}{l}0,36 \\
0,14\end{array}$ & $\begin{array}{l}0,60 \\
0,18\end{array}$ & \multirow{4}{*}{$\begin{array}{c}\text { Temperatur } \\
250\end{array}$} \\
\hline $\begin{array}{l}\text { 15. V. } 1893 \\
17 . " ~ " ~\end{array}$ & $\begin{array}{l}200 \\
200\end{array}$ & $\begin{array}{l}0,6 \\
0,6\end{array}$ & $\begin{array}{l}0,32 \\
0,09\end{array}$ & $\begin{array}{l}0, \overline{2} 3 \\
0,15\end{array}$ & \\
\hline $\begin{array}{l}\text { 15. V. } 1893 \\
\text { 16. " } "\end{array}$ & $\begin{array}{l}500 \\
500\end{array}$ & $\begin{array}{l}0,4 \\
0,6\end{array}$ & $\begin{array}{l}0,50 \\
0,54\end{array}$ & $\begin{array}{l}1,25 \\
0,90\end{array}$ & \\
\hline 16. V. 1893 & 1000 & 0,8 & 1,35 & 1,69 & \\
\hline 17. V. 1893 & 100 & 0,7 & 0,18 & 0,26 & \multirow{4}{*}{$\begin{array}{c}\text { Temperatur } \\
11^{0}\end{array}$} \\
\hline 17. V. 1893 & 200 & 0,5 & 0,18 & $0,36^{\circ}$ & \\
\hline 18. V. 1893 & 500 & 0,5 & 0,32 & 0,64 & \\
\hline 18. V. 1893 & 1000 & 0,7 & 0,54 & 0,79 & \\
\hline
\end{tabular}

Tabelle IV.

Rhythmiseh wechselnde Belastung und Entlastung.

Rana esculenta. Versuchsdauer: 1 Stunde. Fallhöhe des Gewichtes: $4 \mathrm{~mm}$. 'Temperatur: $18-22^{\circ}$.

\begin{tabular}{|c|c|c|c|c|c|}
\hline Datum & $\begin{array}{l}\text { Belastung } \\
\qquad \mathrm{gr}\end{array}$ & $\begin{array}{c}\text { Muskel- } \\
\text { gewicht } \\
\text { gr }\end{array}$ & $\begin{array}{l}\text { Säureb- } \\
\text { beob- } \\
\text { achtet }\end{array}$ & $\begin{array}{l}\text { lung in mgr } \\
\text { reducirt auf } \\
1,0 \text { gr Muskel- } \\
\text { gewicht }\end{array}$ & $\begin{array}{c}100000 \text { grmm } \\
\text { Fallarbeit } \\
\text { geben mgr } \\
\text { Säure }\end{array}$ \\
\hline & \multicolumn{5}{|c|}{ A. 1500 Erschütterangen } \\
\hline & $\begin{array}{l}50 \\
50\end{array}$ & $\begin{array}{l}1,0 \\
0,8\end{array}$ & $\begin{array}{l}0,36 \\
0,54\end{array}$ & $\begin{array}{l}0,36 \\
0,68\end{array}$ & $\begin{array}{l}0,12 \\
0,18\end{array}$ \\
\hline 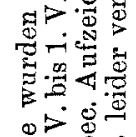 & $\begin{array}{l}100 \\
100\end{array}$ & $\begin{array}{r}0,8 \\
0,8 \\
\text { B. }\end{array}$ & $\begin{array}{c}0,90 \\
0,82 \\
0 \text { Erscl }\end{array}$ & $\begin{array}{r}1,13 \\
1,02 \\
\\
\text { itterungen. }\end{array}$ & $\begin{array}{l}0.15 \\
0.14\end{array}$ \\
\hline 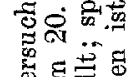 & $\begin{array}{l}50 \\
50\end{array}$ & $\begin{array}{l}0,6 \\
0,8\end{array}$ & $\begin{array}{l}0,92 \\
0,32\end{array}$ & $\begin{array}{l}1,53 \\
0,40\end{array}$ & $\begin{array}{l}0,08 \\
0,03\end{array}$ \\
\hline 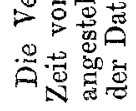 & $\begin{array}{l}100 \\
100\end{array}$ & $\begin{array}{l}0,7 \\
0,9\end{array}$ & $\begin{array}{l}1,27 \\
1,13\end{array}$ & $\begin{array}{l}1,81 \\
1,26\end{array}$ & $\begin{array}{l}0,05 \\
0,05\end{array}$ \\
\hline
\end{tabular}


Beiträge zur Kenntniss der Säurebildung und des Stoffumsatzes etc. 385

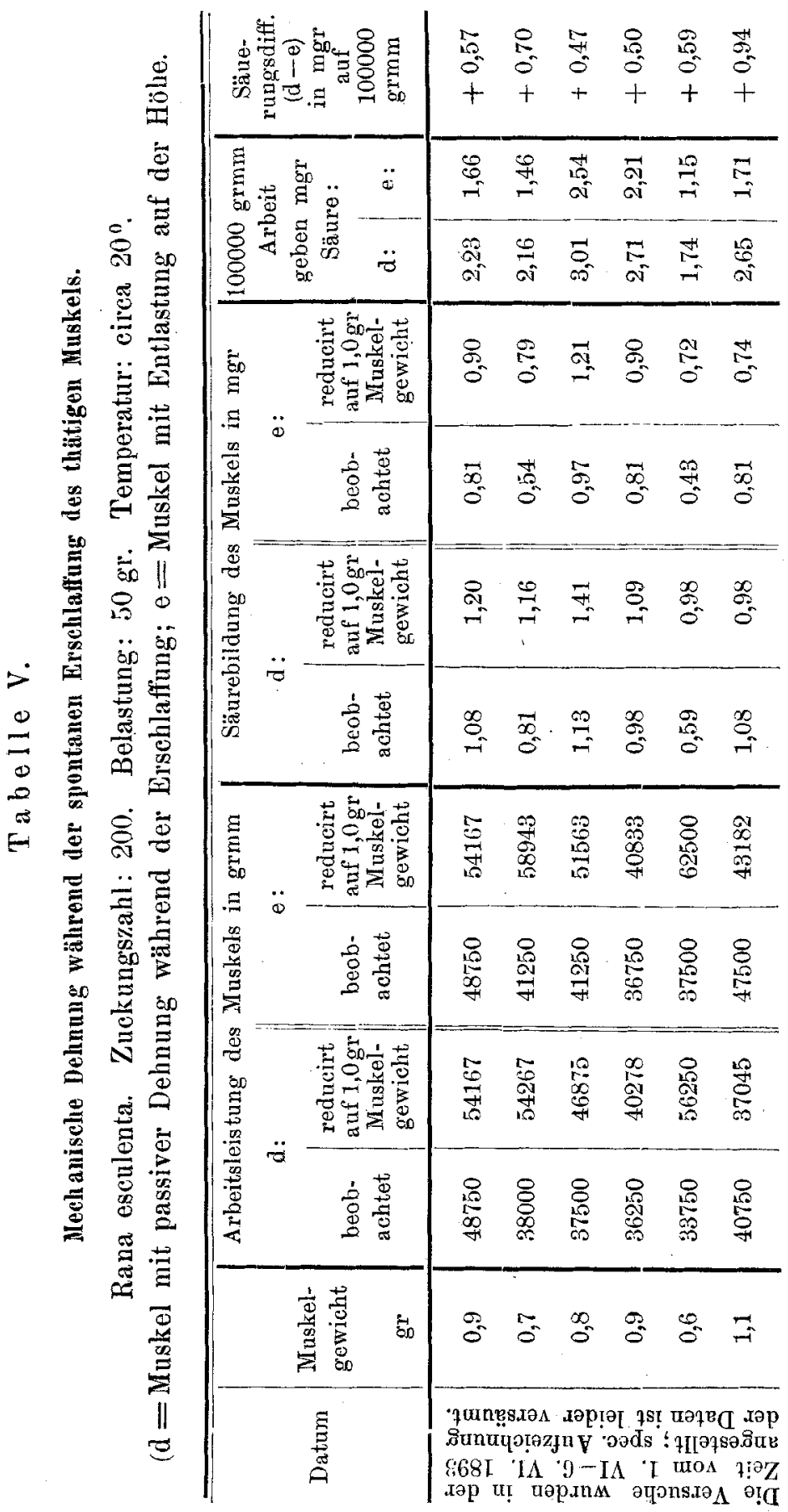




\section{Literatur-Verzeichniss.}

1) Du Bois Reymond, De fibrae muscularis reactione etc. Berolini 1859 .

Monatsber. d. Kgl. Preuss. Akademie d. Wiss. 31. III. 1859.

2) Archiv f. exper. Pathologie und Pharmakologie. Bd. 31. S. 227.

3) R. Heidenhain, Mechanische Leistung. Wärmeentwicklung und Stoffumsatz bei der Muskelthätigkeit. Leipzig 1864.

4) Pfl ü g e r's Archiv f. d. gesammte Physiologie. Bd. 2. S. $423 \mathrm{ff}$.

5) Rianke, Tetanus. Leipzig 1865.

6) Gross, Ueber den Einfluss des Nervensystems auf die Säuerung des Muskels nach dem Tode. Inaug.-Diss. Breslau 1888.

7) Du Bois-Reymond's Archiv f. Physiologie. 1893. Suppl. S. 74 ff.

8) Pflüg e r's Archiv f. d. gesammte Physiologie. Bd. 50. S. $339 \mathrm{ff}$.

9) Ebd. Bd. 50. S. $84 \mathrm{ff}$.

10) Ebd. Bd. 50. S. $551 \mathrm{ff}$.

11) Centralblatt für Physiologie. 1887. S. $195 \mathrm{f}$

12) Archives italiennes; Tome VIII. p. 60 suiv.

13) Meyerhold, Ein Beitrag zur Kenntniss der sauren Reaktion des Muskels. Inaug.-Diss. Erlangen 1892.

14) D u B ois - R e y mond's Archiv f. Physiologie. 1893. S. 423 ff.

14a) Ebd. 1890. Suppl, $59 \mathrm{ff}$.

15) Pflüger's Archiv f. d. ges. Physiologie. Bd. 45. S. $358 \mathrm{f}$.

16) Du Bois-Rey mond's Archiv f. Physiologie. 1880. S. $169 \mathrm{ff}$.

17) P fl üger's Archiv f. d. ges. Physiologie. Bd. 24. S. $229 \mathrm{ff}$.

18) Ebd. Bd. 35. S. $45 \mathrm{ff}$.

19) Ebd. Bd. 39. S. $241 \mathrm{ff}$.

20) Ebd. Bd. 4. S. $57 \mathrm{ff}$.

21) Ebd. Bd. 18. S. $247 \mathrm{ff}$.

22) Ebd. Bd. 16. S. $157 \mathrm{ff}$.

23) Gazette méd. 1875. Nr. 7. (Citirt nach Voi t, Zeițschr. f. Biologie. Bd. 14. S. $118 \mathrm{f}$ )

24) P f l ï ger's Archiv f. d ges. Physiologie. Bd, 4. S. $401 \mathrm{ff}$.

25) D u B o is - R e y m ond's Archiv f. Physiologie. 1876. S. $259 \mathrm{ff}$.

26) Ebd. 1876. S. $567 \mathrm{ff}$.

27) Zeitschrift für Biologie. Bd. 14. S. $135 \mathrm{f}$.

28) Comptes rendus. Tome 48. p. 683.

29) Americ. journ. of the medic. sciences. 46. p. 523. (Citirt nach Hermann, Handbuch der Physiologie I, 1. S. 316.)

30) St. George hospital reports. IIr. p. 149. (Citirt nach Hermann, Handbuch der Pbysiologie 1, 1. S. 316.) 
Beiträge zür Kenntniss der Säurebildung und des Stoffumsatzes etc. 387

31) Pf I ü ge r's Archiv f. d. ges. Physiologie. Bd. 13. S. $626 \mathrm{ff}$.

32) Arch. f. exper. Path. u. Pharm. Bd. 8. S. $422 \mathrm{ff}$.

33) Zcitschrift f. Biologie. Bd. 25. S. $170 \mathrm{ff}$.

34) Deutsches Arch. f. klin. Med. Bd. 10. S. 90. (Citirt nach H e r ma n n, Handbuch der Physiologie I, 4. S. 316.)

35) P fl ü ge r's Archiv f. d.' ges. Physiologie. Bd. 4. S. 495.

36) Wiener med. Wochenschrift. 1853. S. 161 und 1855. S. 681. (Citirt nach Voït (27).)

37) $\mathrm{Ch}$ as anowitz, Ueber den Einfluss des Lichtes auf die Kohlensäureausscheidung etc. Inaug-Diss. Königsberg 1872.

38) Pott, Untersuchungen über die Mengenverhältnisse etc. Habilitationsschrift. Jena 1875.

39) P flüger's Archiv f. d. ges. Physiologie. Bd. 11. S. 289 ff.

40) Zeitschrift für klin. Medicin. Bd. 3. S. $450 \mathrm{ff}$.

41) C r a mer, Ueber das Wesen des Zitterns. Diss. Breslan 1886.

42) Fick, Mechan. Arbeit und Wärmeentwicklung bei der Muskelthätigkeit. Internat. wiss. Bibliothek. Bd. 51. S. 234.

43) P flüger's Archiv f. d. gesammte Physiologie. Bd. 21. S. 239 f.

44) Vierteljahrsschrift der naturforschenden Gesellschaft zu Zürich. XI, $48 \mathrm{ff.}$ Centralblatt f. d. med. Wissenschaften. 1869, S. $611 \mathrm{f}$.

45) Ebd. 1869. S. 17 ff. S. 241 ff. S. 804 ff.

46) Ebd. 1869. S. $161 \mathrm{ff}$.

A. B. M e y e r, Beiträge zur Lehre von der elektr. Nervenreizung. Diss. Zürich 1867.

47) P f lüg er's Archiv f. d. gesammte Physiologie. Bd. 52. S. $592 \mathrm{ff}$. und S. $603 \mathrm{ff}$.

48) Helm oltz, Die Lehre von den Tonempfindungen. S. 600 . Braunschweig 1877.

49) Da B o is-R ey mond, Gesammelte Abhandlungen. Bd. II. S. $191 \mathrm{ff}$.

50) D u B ois-Rey mond's Archiv für Physiologie. 1893.

51) S. 42) and 14a).

52) Fick, Myothermische Untersuchungen. S. auch 42).

58) D u Bois-Reymond's Archiv f. Physiologie. 1880. S. $348 \mathrm{ff.} 1892$. S. 1 ff. u. a. m.

54) P fl ü g e r's Archịv für d. ges. Physiologie. Bd. 50. S. 166 ff. Bd. 51. S. $509 \mathrm{ff}$. Bd. 52. S. $456 \mathrm{ff}$.

55) S o g a 1 । a, Beiträge z. mechan. Analyse d. Muskelzuckungskurven. Inang.Diss. Würzburg 1889.

56) Skandinavisches Archiv f. Physiologie. Bd. I. S. 3 ff.

57) P f l üg e r's Archiv f. d. ges. Physiologie. Bd. 11. S. 196 ff.

58) Ebd. Bd. 25. S. 512 f.

59) Du B ois-Rey mond's Archiv f. Physiologie. 1880. S. 374.

60) P f I üger's Archiv f. d. ges. Physiologie. Bd. 54. S. 162.

61) Poggendorff's Annalen der Physik und Chemie $e_{x}$ Bd. 112. S. $159 \mathrm{f}$. ๒. Phüger, Archiv 1. Physiologie $B a, 56$. 
388 Emil Gotschlich: Beiträge zur Kenntniss der Säurebildnng etc.

62) Archiv f. Anatomie u. Physiologie. Abth. 1878. S. $222 \mathrm{ff}$.

63) Hen le u. P f e u fer, Zeitschrift f. rat. Med. III. Serie. Bd. 20. S. 67.

64) Centralblatt f. d. med. Wissenschaften. 1863. S. 545.

65) Westermann, Ein Beitrag zur Physik des Muskels. Dorpat 1868. (Citirt nach Hermann's Handbuch der Physiologie I, 1.)

66) Centralblatt f. d. med. Wissenschaften. 1867. S. 83.

Comptes rendus. Tome 68, p. 936.

67) Zeitschrift für Biologie. Bd. 21. S. $190 \mathrm{ff}$. - Fi c k, Myotherm, Unters. IX.

68) P flü g e r's Archiv f. d. ges. Physiologie. Bd. 21. - Fi ck, Myotherm. Unters. VIII.

69) D u B o is - R e y mo nd, Gesammelte Abhandlungen. Bd. II. S. 313.

70) P flüger's Archiv f. d. ges. Physiologie. Bd. 53. S. $368 \mathrm{ff}$.

71) Sitzungsberichte der Kgl. sächsischen Akad. d. Wiss. 1871. S. 690 ff.

72) Sitzungsberichte der Kgl. bairischen Akad. d. Wiss. 1861. S. 63.

73) Henle u. Pf eufers Zeitschr. f. rat. Med. III. Serie. Bd. 18. S. $270 \mathrm{ff}$.

74) F. Krause, De rigore mortis etc. Inaug.-Diss. Dorpat 1853. S. 40 ff.

75) Wu ndt, Die Lehre von der Muskelbewegung. S. $71 \mathrm{f}$.

76) Pflüger's Archiv f. die gesammte Physiologie. Bd. 17. S. 282.

77) S. 42) und 52).

78) Pflüger's Archiv für die gesammte Physiologie. Bd. 14. S. 293 ff.

79) Ebd. Bd. 15. S. 89.

80) Zeitschrift für klinische Medicin. 1890. Jubelsuppl. S. 70.

81) Pfl ü ger's Archiv für die gesammte Physiologie. Bd. 10. S. 317. 